\title{
Timpanometria em lactentes utilizando sonda multifrequencial
}

\section{Multifrequency tympanometry in infants}

\author{
Tamyne Ferreira Duarte de Moraes', Camila de Cássia Macedo², Mariza Ribeiro Feniman³.
}

1) Mestre em Ciências. Fonoaudióloga residente do Programa de Residência Multiprofissional do Hospital de Reabilitação de Anomalias Craniofaciais - Universidade de São Paulo.

2) Mestre em Ciências da Reabilitação. Doutoranda do Programa de Pós-graduação do Hospital de Reabilitação de Anomalias Craniofaciais - Universidade de São Paulo. 3) Professora Titular do Departamento de Fonoaudiologia FOB/USP.

Instituição: Faculdade de Odontologia de Bauru - Universidade de São Paulo. Bauru / SP - Brasil.

Endereço para correspondência: Tamyne Ferreira Duarte de Moraes - Rua Espírito Santo 4-63 - Vila Coralina - Bauru / SP - Brasil - CEP: 17030-030 - E-mail: tamyne.fono@gmail.com

Dissertação realizada com financiamento da Coordenação de Aperfeiçoamento de Pessoal de Nível Superior.

Artigo recebido em 27 de junho de 2011. Artigo aprovado em 6 de fevereiro de 2012.

\section{RESUMO}

Introdução: O uso de uma única frequência na timpanometria não é sensível na detecção de todos os casos de alteração na orelha média, dificultando o diagnóstico preciso.

Objetivo: Caracterização das medidas de imitância acústica de lactentes utilizando três tipos de sonda. Estudo prospectivo. Método: Foram avaliados 54 lactentes, com idade entre zero e três meses. Os critérios de inclusão foram ausência de infecções de vias aéreas, presença de emissões otoacústicas evocadas transientes, ausência de indicadores de risco para perda auditiva. Foi realizada entrevista audiológica, inspeção visual do meato acústico externo e medidas de imitância acústica nas frequências de $226 \mathrm{~Hz}, 678 \mathrm{~Hz}$ e $1000 \mathrm{~Hz}$. Foram coletados os registros timpanométricos de efeito de oclusão, curva e pressão de pico timpanométrico, volume equivalente do meato acústico externo e pico compensado da admitância acústica estática.

Resultados: Os resultados indicaram presença de efeito de oclusão $(2,88 \%$ em $226 \mathrm{~Hz}, 4,81 \%$ em $678 \mathrm{~Hz}$ e $3,85 \%$ em $1000 \mathrm{~Hz})$; predomínio de curva em pico único $(65,35 \% \mathrm{em}$ $226 \mathrm{~Hz}, 81,82 \%$ em $678 \mathrm{~Hz}$ e $77,00 \%$ em $1000 \mathrm{~Hz}$ ); pressão de pico variando de -150 a $180 \mathrm{daPa}$; aumento do volume equivalente do meato acústico externo com aumento da frequência da sonda $(0,64 \mathrm{ml} \mathrm{em} 226 \mathrm{~Hz}, 1,63 \mathrm{mmho}$ em $678 \mathrm{~Hz}$ e $2,59 \mathrm{mmho}$ em $1000 \mathrm{~Hz}$ ); aumento do pico compensado da admitância acústica estática $(0,51 \mathrm{ml}$ em $226 \mathrm{~Hz}, 0,55 \mathrm{mmho}$ em $678 \mathrm{~Hz}$ e $1,20 \mathrm{mmho}$ em $1000 \mathrm{~Hz}$ ). Foram classificados como normais $93,06 \%$ dos timpanogramas com $226 \mathrm{~Hz}, 80,81 \%$ em $678 \mathrm{~Hz}$ e $82,00 \%$ em $1000 \mathrm{~Hz}$.

Conclusão: Por meio destas avaliações e resultados foi possível caracterizar as medidas de imitância acústica dos lactentes. Palavras-chave: audição, lactente, orelha média, testes de impedância acústica.

\section{SUMMARY}

Introduction: The use of conventional tympanometry is not sufficiently sensitive to detect all cases of middle ear changes, and this hinders accurate diagnosis.

Objective: To characterize acoustic immittance measures of infants from 0 to 3 months of age using multifrequency tympanometry in a prospective study.

Method: 54 infants from 0 to 3 months of age were evaluated. The inclusion criteria included absence of respiratory infections during the evaluation, presence of transient evoked otoacoustic emissions, and absence of risk indicators for hearing loss. The subjects were evaluated by an audiologic interview, a visual inspection of the ear canal, and measures of acoustic immittance at the frequencies of $226 \mathrm{~Hz}, 678 \mathrm{~Hz}$, and 1,000 $\mathrm{Hz}$. Tympanometric records of the occlusion effect, tympanometric curve type, tympanometric peak pressure, equivalent ear canal volume, and peak compensated static acoustic admittance were collected.

Results: The results indicated the presence of an occlusion effect $(2.88 \%$ at $226 \mathrm{~Hz}, 4.81 \%$ at $678 \mathrm{~Hz}$ and $3.85 \%$ at 1,000 $\mathrm{Hz}$ ), predominance of a tympanometric curve with a single peak $(65.35 \%$ at $226 \mathrm{~Hz}, 81.82 \%$ at $678 \mathrm{~Hz}$, and $77.00 \%$ at 1,000 $\mathrm{Hz}$ ), and tympanometric peak pressure ranging from -155 to $180 \mathrm{daPa}$. Further, the equivalent ear canal volume increased with the frequency of the probe $(0.64 \mathrm{~mL}$ at $226 \mathrm{~Hz}, 1.63 \mathrm{mho}$ at $678 \mathrm{~Hz}$, and $2.59 \mathrm{mmho}$ at $1,000 \mathrm{~Hz}$ ) and the peak compensated static acoustic admittance values increased with an increase in frequency $(0.51 \mathrm{~mL}$ at $226 \mathrm{~Hz}, 0.55 \mathrm{mmho}$ at 678 $\mathrm{Hz}$ and $1.20 \mathrm{mmho}$ at 1,000 Hz). 93.06\% of the tympanograms were classified as normal at $226 \mathrm{~Hz}, 81.82 \%$ at $678 \mathrm{~Hz}$, and $77.00 \%$ at $1,000 \mathrm{~Hz}$, respectively.

Conclusion: Taken together, these results demonstrated that utilizing these evaluations made it possible to characterize the acoustic immittance measures of infants.

Keywords: hearing, infant, middle ear, acoustic impedance tests. 


\section{INTRODUÇÃO}

A audição é a principal fonte para a aquisição das habilidades de linguagem e fala na criança com audição normal. Uma perda auditiva pode provocar danos nestas habilidades, dificultando o processo de comunicação. Assim, na avaliação audiológica de lactentes, recomenda-se a realização de uma avaliação auditiva global, com medidas eletrofisiológicas, eletroacústicas e com métodos comportamentais.

A medida de imitância acústica é um instrumento eletroacústico valioso na detecção das alterações de orelha média, devido a sua rapidez e objetividade. Caracteriza-se pela análise de respostas mecânicas do sistema auditivo em resposta à estimulação acústica e se relaciona com a transferência da energia acústica que ocorre quando as ondas sonoras chegam até o meato acústico externo, quando se aplica uma pressão sonora sobre a membrana timpânica, provocando a movimentação desta. Esta medida se refere à facilidade ou a oposição a este fluxo de energia sonora dentro do sistema auditivo.

A timpanometria com multifrequência surgiu como promessa de um novo método de avaliação das condições da orelha média, sendo um método rápido, fácil, não invasivo, objetivo e apresentando maior sensibilidade que a timpanometria convencional, realizada na frequência de 226Hz, pois episódios de Otite Média Aguda nem sempre são detectados na timpanometria convencional (1).

Todas as patologias de orelha média que são identificadas por meio da timpanometria convencional $(226 \mathrm{~Hz})$ podem ser sempre identificadas pela timpanometria de multifrequência. Porém, a timpanometria convencional pode falhar na detecção de patologias de orelha média que são corretamente identificadas pela timpanometria de multifrequência, pois esta é capaz de identificar pequenas alterações no mecanismo acústico da orelha média. Alguns autores recomendaram a combinação da timpanometria convencional com a de multifrequência em neonatos, crianças e adultos (2-5).

Até os dois anos de idade, o sistema tímpanoossicular apresenta um comportamento diferente, tendo como característica física dominante o fator massa, que pode ser avaliado com maior eficiência por alta frequência, como $678 \mathrm{~Hz}$ e $1000 \mathrm{~Hz}$. Após esse período ocorre uma mudança no comportamento, chegando à fase adulta dominado pelo fator rigidez, avaliada pela frequência de 226Hz (6).

Variados estudos (7-10) utilizam timpanometria na frequência de $226 \mathrm{~Hz}$ na avaliração de lactentes. Porém a literatura mostra que o uso de uma única frequência não é sensível o suficiente na detecção de todos os casos, o que dificulta o diagnóstico preciso (11-14).

As medidas de imitanciometria em alta frequência ajudam a esclarecer resultados falso-positivos na triagem que ocorrem devido à patologia na orelha média ou presença de secreção. A avaliação adequada das condições da orelha média no período neonatal resulta em encaminhamentos adequados à equipe médica e audiológica, podendo levar à melhora da eficácia dos programas de triagem auditiva neonatal (15).

Assim, sabe-se que o uso de uma única frequência de sonda nas medidas de imitância acústica não é sensível o suficiente na caracterização de todos os casos de alteração na orelha média, dificultando o diagnóstico preciso.

Portanto, o objetivo deste trabalho foi realizar a caracterização das medidas de imitância acústica de lactentes com idade entre zero e três meses de idade, utilizando três tipos de sonda.

\section{MÉTODO}

Este trabalho foi aprovado pelo Comitê de Ética em Pesquisa da instituição onde foi realizado (protocolo número 52/2009). Selecionados os lactentes, os pais/responsáveis receberam um termo de consentimento livre e esclarecido, contendo informações sobre a pesquisa, em linguagem clara e simples. Somente após a concordância dos mesmos e assinatura do termo é que foram iniciadas as avaliações.

Foram definidos como critério de inclusão no estudo a idade do lactente (variando entre zero e três meses), ausência de infecções das vias aéreas superiores no dia da avaliação proposta, meato acústico externo sem impedimentos para realização do exame, presença de Emissões Otoacústicas Evocadas Transientes no dia da avaliação, ausência de indicadores de risco para perda auditiva definidos pelo Joint Committee on Infant Hearing(16).

Seguindo estes critérios de inclusão, foram avaliados 54 lactentes, sendo $27(50,0 \%)$ do gênero feminino e 27 $(50,0 \%)$ do masculino. A idade dos lactentes variou de 8 a 115 dias, com idade média correspondente a 31,8 dias.

Em quatro lactentes não foi possível avaliar ambas as orelhas. Portanto, foram avaliadas 104 orelhas, sendo 51 $(49,1 \%)$ direitas e $53(50,9 \%)$ esquerdas. O processo de avaliação constituiu de entrevista audiológica, inspeção visual do meato acústico externo e avaliação das medidas de imitância acústica. 
A entrevista audiológica foi realizada com os pais/ responsáveis por meio de perguntas dirigidas, em linguagem acessível, com o objetivo de obter informações sobre a audição do lactente, presença de infecção de vias aéreas superiores e de indicadores de risco para perda auditiva.

A inspeção visual do meato acústico externo foi realizada por uma fonoaudióloga com o objetivo de verificar a presença de algum impedimento para realização das medidas de imitância acústica. Foi utilizado um otoscópio de marca Heine e espéculos esterilizados. Na presença de algum impedimento para realização das avaliações (como, por exemplo, a presença de cera), o lactente foi encaminhado para avaliação otorrinolaringológica.

Para obtenção das medidas de imitância acústica foi utilizado o equipamento GSITympStarversão 2, Middle Ear Analyzer, marca Grason-Stadler, e realizadas as Medidas de Imitância Acústica com tom de sonda de $226 \mathrm{~Hz}, 678 \mathrm{~Hz}$ e $1000 \mathrm{~Hz}$. A imitância acústica foi realizada para cada frequência com variação de pressão de ar de +200daPa a -400daPa, com velocidade de variação de pressão automática de 600/ 200daPa por segundo. A intensidade do tom na sonda foi 85 dBNPS para $226 \mathrm{~Hz}$ e $678 \mathrm{~Hz}$,e 75 dBNPS em $1000 \mathrm{~Hz}$, conforme orientações do manual equipamento (17).

Foram coletados 300 timpanogramas, sendo 101 $(33,67 \%)$ correspondentes ao tom de sonda de $226 \mathrm{~Hz}, 99$ $(33,00 \%)$ correspondentes ao tom de sonda de $678 \mathrm{~Hz}$ e $100(33,33 \%)$ referentes ao tom de sonda de $1000 \mathrm{~Hz}$. A distribuição dos timpanogramas por tom de sonda analisado não foi igual devido à presença de efeito de oclusão em algumas orelhas, conforme descrito posteriormente.

Foram definidos para análise do timpanograma os parâmetros Efeito de Oclusão, Forma da Curva Timpanométrica, Pressão de Pico Timpanométrico, Volume Equivalente do Meato Acústico Externo, Pico Compensado de Admitância Acústica Estática.

O Efeito de Oclusão define-se como o registro automático do aparelho diante da impossibilidade de registrar os dados timpanométricos. Diante da presença deste efeito no tom de sonda de $226 \mathrm{~Hz}$, foi retirada a oliva da orelha do lactente e reposicionada, sendo reiniciado o exame. Na presença do efeito de oclusão nas demais frequências $(678 \mathrm{~Hz}$ e $1000 \mathrm{~Hz})$, foi realizada uma nova tentativa de obtenção do timpanograma na frequência testada, após refazer o exame em 226Hz.

A Forma da Curva Timpanométrica foi classificada em curva timpanométrica em pico único (PU), com presença de um pico de admitância máxima; curva timpanométrica em pico duplo (PD), com presença de dois picos de admitância; curva timpanométrica assimétrica
(AS), com decréscimo de admitância gradual, com variação de+ 200 para - 200daPa; curva timpanométrica em pico invertido (IN), com presença de pico de admitância invertido em relação à $+200 \mathrm{daPa}$ e $-200 \mathrm{daPa}$; curva timpanométrica Plana (PL), com ausência de pico de admitância.

A Pressão de Pico Timpanométrico (PPT) é a medida da pressão do pico de máxima admitância, expresso em daPa. O Volume Equivalente do Meato Acústico Externo (Vea) é a medida da admitância acústica registrada em $+200 \mathrm{daPa}$, expressa em $\mathrm{ml}$ para $226 \mathrm{~Hz}$ e em mmho nas frequências de $678 \mathrm{~Hz}$ e $1000 \mathrm{~Hz}$. Pico Compensado de Admitância Acústica Estática (Ymt) se refere ao valor do pico de máxima admitância do sistema tímpano-ossicular, no qual a pressão da orelha externa e da orelha média se iguala. Valores mensurados em $\mathrm{ml}$ para $226 \mathrm{~Hz}$ e em mmho nas frequências de $678 \mathrm{~Hz}$ e $1000 \mathrm{~Hz}$ mmho.

$\mathrm{Na}$ frequência de $226 \mathrm{~Hz}$ foram adotados como normais os timpanogramas que apresentaram curva timpanométrica em pico único ou em pico duplo, de acordo com outros estudos $(19,28)$. As frequências de $678 \mathrm{~Hz}$ e $1000 \mathrm{~Hz}$ foram analisadas utilizado o protocolo de Sutton (18), recomendado para análise timpanométrica de lactentes com idade até quatro meses. Seguindo este protocolo, foram classificados como normais os timpanogramas que apresentavam Ymt $>0$ e PPT> 200daPa. Os timpanogramas classificados como anormais apresentavam Ymt $\leq 0$ ou PPT $<-200 \mathrm{daPa}$.

De acordo com a classificação dos timpanogramas foram feitas as análises dos resultados timpanométricos dos lactentes, sendo realizadas as comparações entre os tipos de sonda dos timpanogramas classificados como normais. Também foram realizadas análises descritivas dos resultados dos timpanogramas classificados como normais por meio de tabelas e gráficos, além das demais análises feitas por testes estatísticos.

Para comparar os dados do PPT, Vea e Ymt nas três diferentes frequências testadas foi utilizado um teste não paramétrico, pois a amostra não apresentou distribuição normal. Assim, utilizou-se o teste Qui-Quadrado e o Teste de Friedman, adotando como nível de significância $p=0,05$.

Foram realizadas comparações individuais entre os valores de PPT, Vea e Ymt nas frequências avaliadas. Para realizar estas comparações foi utilizado o Teste de Friedman, sendo que foi possível a realização apenas nos sujeitos que apresentavam timpanogramas normais em todas as frequências testadas, ou seja, para esta análise foram excluídos os sujeitos que nãoapresentaram timpanogramas normais em todas as frequências testadas. Assim, para esta análise o número de sujeitos foi 68. 


\section{RESULTADOS}

O efeito de oclusão esteve presente nas três frequências $(226 \mathrm{~Hz}, 678 \mathrm{~Hz}$ e $1000 \mathrm{~Hz})$. Na Tabela 1 está indicada a ocorrência deste efeito considerando o número total de orelhas avaliadas $(\mathrm{N}=104)$.

Foram coletados 300 timpanogramas, sendo 101 com tom de sonda de $226 \mathrm{~Hz}, 99 \mathrm{com}$ tom de sonda de $678 \mathrm{~Hz}$ e $100 \mathrm{com}$ tom de sonda de $1000 \mathrm{~Hz}$. No Gráfico 1 está representada a distribuição da ocorrência da forma da curva timpanométrica por tom de sonda avaliado.

Foi realizada uma comparação dos valores percentuais da forma da curva timpanométrica e do efeito de oclusão das três frequências, considerando a variável gênero e idade, conforme Tabela 2.

Os resultados de PPT, Vea e Ymt foram analisados de acordo com a frequência analisada, considerando apenas os timpanogramas que foram classificados como normais. A Tabela 3 descreve os valores de PPT encontrados nas orelhas avaliadas.

A análise de PPT por meio do Teste Qui-Quadrado indicou diferença significante entre as frequências testadas $(\mathrm{p}<0,00003)$. Assim, comparando individualmente as frequências por meio do Teste de Friedman, verificou-se que os valores médios obtidos com sonda de $1000 \mathrm{~Hz}$ foram superiores aos valores médios com sonda de $678 \mathrm{~Hz}$,

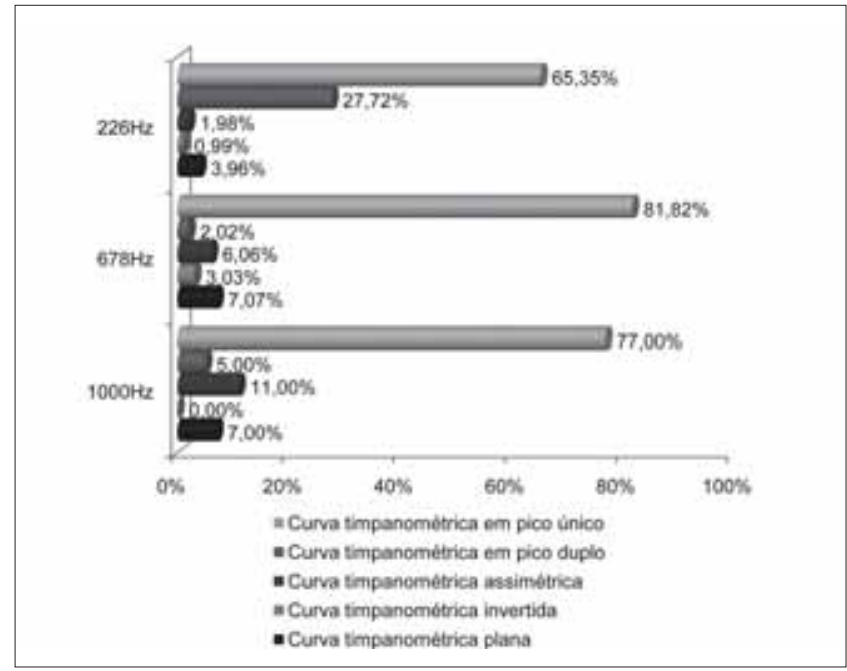

Gráfíco 1. Ocorrência de forma da curva timpanométrica por frequência.

Tabela I. Ocorrência de efeito de oclusão por frequência

\begin{tabular}{lcc}
\hline & \multicolumn{2}{c}{ Efeito de Oclusão } \\
& $N$ & $\%$ \\
\hline $226 \mathrm{~Hz}$ & 3 & 2,88 \\
$678 \mathrm{~Hz}$ & 5 & 4,81 \\
$1000 \mathrm{~Hz}$ & 4 & 3,85 \\
\hline Total & 12 & $11,54 \%$ \\
\hline
\end{tabular}

Tabela 2. Ocorrência do efeito de oclusão e da forma da curva timpanométrica segundo o gênero e idade.

\begin{tabular}{ccccccc}
\hline Achadostimpanométricos & \multicolumn{2}{c}{ Gênero } & \multicolumn{2}{c}{ Idade } \\
& Masculino & Feminino & 8 a 29 dias & 30 a 59 dias & 60 a 89 dias & 90 a I I5 dias \\
& $N=156(\%)$ & $N=156(\%)$ & $N=255(\%)$ & $N=18(\%)$ & $N=15(\%)$ & $N=24(\%)$ \\
\hline PU & 66,67 & 76,28 & 70,20 & 61,11 & 86,67 & 79,17 \\
PD & 12,82 & 9,62 & 10,98 & 16,67 & & 12,50 \\
AS & 7,69 & 6,41 & 7,06 & 11,11 & & 4,17 \\
IN & 0,64 & 1,92 & 0,78 & 5,56 & & 4,17 \\
PL & 5,13 & 5,13 & 5,49 & 5,56 & 6,67 & 0 \\
EO & 7,05 & 0,64 & 4,31 & 0,00 & 6,67 & 0 \\
\hline
\end{tabular}

Legenda: N - Número de orelhas avaliadas; PU - curva timpanométrica em pico único; PD - curva timpanométrica em pico duplo; AS - curva timpanométrica assimétrica; IN - curva timpanométrica invertida; PL - curva timpanométrica plana; EO - efeito de oclusão.

Tabela 3. Valores de pressão de pico timpanométrico.

\begin{tabular}{lccccccc}
\hline Frequência & $N^{\circ}$ deorelhas & $\begin{array}{c}\text { Média } \\
(\mathrm{daPa})\end{array}$ & $\begin{array}{c}\text { Desvio-padrão } \\
(\mathrm{daPa})\end{array}$ & $\begin{array}{c}\text { Mínimo } \\
(\mathrm{daPa})\end{array}$ & $\begin{array}{c}\text { Máximo } \\
(\mathrm{daPa})\end{array}$ & $1^{\circ}$ & $\begin{array}{c}\text { Quartil } \\
3^{\circ}\end{array}$ \\
\hline $226 \mathrm{~Hz}$ & 94 & 27,93 & 39,10 & -65 & 115 & 6,25 & 53,75 \\
$678 \mathrm{~Hz}$ & 81 & 24,75 & 55,35 & -150 & 145 & 0 & 57,50 \\
$1000 \mathrm{~Hz}$ & 82 & 36,49 & 59,76 & -115 & 180 & 6,25 & 60,00 \\
\hline
\end{tabular}


Tabela 4. Valores de volume equivalente do meato acústico externos.

\begin{tabular}{llllllll}
\hline & \multicolumn{10}{c}{$1^{\circ}$} & \multicolumn{1}{c}{$3^{\circ}$} \\
\hline $226 \mathrm{~Hz}$ & 94 & 0,64 & 0,24 & 0,20 & 1,60 & 0,50 & 0,70 \\
$678 \mathrm{~Hz}$ & 81 & 1,63 & 0,74 & 0,60 & 4,80 & 1,20 & 1,80 \\
$1000 \mathrm{~Hz}$ & 82 & 2,59 & 0,92 & 1,00 & 5,80 & 2,00 & 2,90 \\
\hline
\end{tabular}

Tabela 5. Valores de pico compensado de admitância acústica estática.

\begin{tabular}{lccccccc}
\hline Frequência & Nodeorelhas & Média & Desvio-padrão & Mínimo & Máximo & \multicolumn{2}{c}{ Quartil } \\
& & & & & & $1^{\circ}$ & $3^{\circ}$ \\
\hline $266 \mathrm{~Hz}$ & 94 & 0,51 & 0,32 & 0,10 & 1,60 & 0,30 & 0,70 \\
$678 \mathrm{~Hz}$ & 81 & 0,55 & 0,47 & 0,10 & 2,30 & 0,20 & 0,80 \\
$1000 \mathrm{~Hz}$ & 82 & 1,20 & 1,13 & 0,10 & 5,90 & 0,40 & 1,50 \\
\hline
\end{tabular}

sendo este último também superior aos valores de $226 \mathrm{~Hz}$. Esta diferença foi estatisticamente significante.

Com relação aos dados de Vea, descritos na Tabela 4, o Teste Qui-Quadrado indicou presença de diferença significante entre os resultados, sendo $\mathrm{p}<0,0000$.

O teste de Friedman apontou que houve diferença significante na comparação individual entre as médias de Vea nas frequências analisadas, sendo os valores médios com tom de sonda de $1000 \mathrm{~Hz}$ superiores aos valores com $678 \mathrm{~Hz}$, assim como valores médios com sonda de $1000 \mathrm{~Hz}$ superiores aos valores com $226 \mathrm{~Hz}$. A Tabela 5 apresenta as medidas de Ymt obtidas nas frequências avaliadas,além da análise estatística descritiva.

O teste Qui-Quadrado indicou que houve diferença significante na comparação dos resultados $(p<0,0000)$. As comparações individuais entre as frequências realizada por meio do Teste de Friedman mostrou que houve diferença significante entre os valores médios obtidos, sendo os valores com sonda de $1000 \mathrm{~Hz}$ superiores aos valores de $678 \mathrm{~Hz}$, assim como valores médios de $1000 \mathrm{~Hz}$ superiores a $226 \mathrm{~Hz}$.

A análise dos timpanogramas permitiu concluir que 93,06\% (94) das orelhas analisadas com tom de sonda de $226 \mathrm{~Hz}$ estavam normais. Com sonda de $678 \mathrm{~Hz}, 80,81 \%$ (80) das orelhas foram classificadas como normais. Já com sonda de $1000 \mathrm{~Hz}$, a porcentagem de orelhas classificadas como normais foi de $82,00 \%$ (82).

\section{DISCUSSÃO}

As orelhas direita e esquerda foram agrupadas para favorecer uma melhor análise dos resultados e caracterização da população amostrada, já que não foi encontrada diferença entre as orelhas nos demais estudos.
A ausência de captação de registro timpanométrico (efeito de oclusão) é associada com diversos fatores: presença de cerúmen na orelha externa, colocação errônea da sonda na orelha do lactente (17) ou devidoàs diferenças existentes entre as características do sistema tímpanoossicular dos adultos e de lactentes. Estas diferenças ocorrem porque nos lactentes o sistema sofre maior influência do fator massa, enquanto que nos adultos é predominante o fator rigidez $(13,22,23)$ e esta diferença pode ser captada pelo analisador de orelha média como oclusão.

Apesar dos cuidados tomados, verificou-se presença de efeito de oclusão nas três frequências analisadas, o que foi discordante da literatura, pois não foi relatado este efeito na timpanometria convencional (19-21). Outro fator discordante foi a maior ocorrência deste efeito em outros estudos ( $49 \%$ em $678 \mathrm{~Hz}$ e $51 \%$ em $1000 \mathrm{~Hz}$ (19); $29 \%$ em $1000 \mathrm{~Hz}$ (20); 97,67 em $678 \mathrm{~Hz}$ e 2,33\% em $1000 \mathrm{~Hz}$ (21)), quando comparados a este.

O efeito de oclusão está presente em lactentes com idade inferior a quatro meses (19). Observou-se maior ocorrência deste efeito em lactentes de até dois meses de vida, estando ausente em lactentes com três meses de vida.

Na timpanometria realizada em $226 \mathrm{~Hz}$ é predominante a ocorrência de curva timpanométrica em pico único, tanto em estudos nacionais (19,21,24,) como internacionais (25). Outros estudos indicam predomínio de curva timpanométrica em pico duplo $(26,28)$.

Apesar de não haver indícios de alterações de orelha média nos lactentes, houve baixa ocorrência de curva timpanométrica plana, o que pode indicar possível presença de fluido na orelha média.

Na frequência de $678 \mathrm{~Hz}$ houve maior discordância com a literatura, que apresentou maior ocorrência de curva 
timpanométrica em pico único $(19,21)$, seguido de curva timpanométrica plana(21), além de outro estudo que indicou maior ocorrência de curva timpanométrica assimétrica(28). Já em $1000 \mathrm{~Hz}$, houve maior ocorrência de curva timpanométrica em pico único(19,21,24,26,28-30).

As diferenças encontradas na forma da curva timpanométrica entre os estudos analisados podem ser decorrentes da variação normal existente entre a população e também pelas diferenças entre a idade dos lactentes.

Curvas timpanométricas em pico único e pico duplo são consideradas normais pela literatura, enquanto as curvas timpanométricas assimétricas, invertidas e planas são consideradas anormais $(18,19,25,28,31)$.

Analisando os resultados, observou-se que a média da pressão de pico timpanométrico (PPT) em $226 \mathrm{~Hz}$ foi menor do que a média em $1000 \mathrm{~Hz}$, assim como em outros estudos $(8,12,19,20,25,26,28)$. Entretanto, os valores encontrados foram superiores aos demonstrados pela literatura. A análise desta variável em $678 \mathrm{~Hz}$ foi realizada por apenas um estudo(19), sendo a média descrita menor do que a média encontrada aqui.

Apesar de haver diferença significante entre as frequências com relação a PPT, não houve diferença clinicamente significante, ou seja, os resultados encontrados nas três frequências estão dentro dos padrões definidos como normais (18).

Valores de PPT inferiores a -100daPa em lactentes caracteriza uma disfunção tubária ou pode ser precursor de otite média secretora $(33,34)$, porém não impede o registro das EOE-t(35).

A medida do volume da orelha externa (Vea) é útil para determinar precisamente a admitância acústica estática compensada e auxiliar na identificação das causas da ocorrência de curvas timpanométricas planas(40). A média de Vea em $226 \mathrm{~Hz}$ foi próxima dos resultados descritos na literatura $(19,28,20,26)$, porém superior a outro estudo(32). Para este tom de sonda, a faixa de normalidade está entre 0,3 e $1,0 \mathrm{ml}(35-37)$.

Com tom de sonda de $678 \mathrm{~Hz}$ a média de Vea também se aproximou dos valores da literatura (19). Em $1000 \mathrm{~Hz}$, a literatura apresenta grande variabilidade, sendo apresentados valores superiores $(25)$ e inferiores $(20,38)$ ao deste estudo, sendo a média em torno de $1,5 \mathrm{mmho}$ $(12,19,26,32)$.

Grason-STadler(39) afirmou que o valor de Vea obtido na frequência de $678 \mathrm{~Hz}$ é três vezes maior do que o valor de Vea em $226 \mathrm{~Hz}$; em $1000 \mathrm{~Hz}$ esta diferença pode chegar em até 4,4 vezes. Tendo em vista estas especificações, pode-se concluir que, embora os valores não se aproximem da literatura, ainda se encontram dentro da normalidade. Concordando com esta afirmação, encontrou-se diferença significante entre os resultados da Vea nas três frequências, sendo a média com tom de sonda de $1000 \mathrm{~Hz}$ maior que $678 \mathrm{~Hz}$ e $226 \mathrm{~Hz}$.

Por meio da medida do pico compensado de admitância acústica estática (Ymt) é possível identificar alterações na orelha média, como presença de secreção, fixação da cadeia ossicular, otosclerose, disjunção de cadeia, dentre outros(34).

Assim como nos valores de Vea, foi observado um aumento gradativo da Ymt na alta frequência, com diferença significante entre a frequência de $1000 \mathrm{~Hz}$ e as demais. A literatura estudada também apresentou média superior de Ymt em $1000 \mathrm{~Hz}$, quando comparada com $226 \mathrm{~Hz}$. $(19,20,26,28)$

Como visto na análise das demais variáveis, apenas um estudo(19) utilizando a frequência de $678 \mathrm{~Hz}$ foi encontrado, indicando valores próximos aos aqui descritos.

Na frequência de $1000 \mathrm{~Hz}$ diversos estudos apresentaram valores de Ymt próximos aos descritos $(12,15,25)$. O estudo mais recente encontrado foi de $\operatorname{Mazlan}(41)$, que ao avaliarem neonatos, encontraram média de Ymt igual a $1,06 \mathrm{mmho}$.

Por meio da análise dos timpanogramas seguindo o protocolo de SutTon(18) obteve-se 93\% de orelhas com timpanogramas normais na frequência de $226 \mathrm{~Hz}$; $81 \%$ de orelhas com timpanogramas normais em $678 \mathrm{~Hz} ; 82 \%$ de orelhas com timpanogramas normais em $1000 \mathrm{~Hz}$, , resultados próximos aos de outros trabalhos $(19,21,28,29,42,43)$.

As orelhas com timpanogramas classificados como anormais apresentaram resultados positivos na avaliação das EOE-t, o que não era esperado. Segundo a literatura, a presença de uma leve disfunção da orelha média, um atraso na maturação do sistema da orelha média dos neonatos, a frequência do tom de sonda não ser alta o suficiente para alguns recém-nascidos, ocorrência de vedação inadequada da sonda ou ainda presença de artefatos de movimento são justificativas para este fato (25).

Outra justificativa disponível na literatura é que a timpanometria de alta frequência parece fornecer informações mais detalhadas sobre o estado de mecânica e acústica da orelha, principalmente em alterações relacionadas ao fator massa $(44,45)$, além da possibilidade de presença de patologia da orelha média em estágio inicial ou final, de 
maneira que não causasse interferência no resultado das $\operatorname{EOE}(43)$.

Estudos $(13,44)$ que avaliaram a sensibilidade e a especificidade da timpanometria indicaram que a sensibilidade da timpanometria convencional é maior que da timpanometria com alta frequência, sendo a especificidade da alta frequência maior que da timpanometria convencional.

Diante das análises e comparações realizadas, é possível inferir que as variáveis analisadas seguem de acordo com os achados da literatura, podendo servir como dados normativos. Entretanto fica evidente que é necessária a continuação da definição de valores normativos em diferentes idades para que seja possível adequar a prática clínica ao uso de tom de sonda de alta frequência em crianças.

\section{CONCLUSÃO}

Por meio das avaliações propostas foi possível realizar a caracterização das medidas de imitância acústica dos lactentes avaliados, conforme descrito abaixo:

- O efeito de oclusão a presentou baixa ocorrência e foi observado nas frequências de $226 \mathrm{~Hz}, 678 \mathrm{~Hz}$ e $1000 \mathrm{~Hz}$;

- A forma da curva timpanométrica em pico único foi predominante nas três frequências avaliadas, sendo $65,35 \%$ em $226 \mathrm{~Hz}, 81,82 \%$ em $678 \mathrm{~Hz}$ e $77,00 \%$ em $100 \mathrm{~Hz}$;

- A pressão de pico timpanométrico apresentou valores médios de $27 \mathrm{daPa}$ em $226 \mathrm{~Hz}, 24 \mathrm{daPa}$ em $678 \mathrm{~Hz}$ e $36 \mathrm{daPa}$ em 1000Hz;

- O volume equivalente do meato acústico externo apresentou valores médios de 0,64ml em $226 \mathrm{~Hz}$, $1,63 \mathrm{mmho}$ em $678 \mathrm{~Hz}$ e $2,59 \mathrm{mmho}$ em $1000 \mathrm{~Hz}$;

- O pico compensado de admitância acústica estática apresentou valores médios de $0,51 \mathrm{ml}$ em $226 \mathrm{~Hz}$, $0,55 \mathrm{mmho}$ em $678 \mathrm{~Hz}$ e $1,20 \mathrm{mmho}$ em $1000 \mathrm{~Hz}$.

\section{REFERÊNCIAS BIBLIOGRÁFICAS}

1. Ferekidis E, Vlachou S, Douniadakis D, Apostolopoulos $\mathrm{N}$, Adamopoulos G. Multiple-frequency tympanometry in children with acute otitis media. Otolaryngol Head Neck Surg. 1999, 121(6):797-801.

2. American Speech-Language-Hearing Association. Working group on aural acoustic-immittance measurements committe on audiologic evaluation.Tympanometry. J Speech Hear Disord. 1988, 53(4):354-377.

3. Johnson KC. Audiologic assessment of children with suspected hearing loss. Otolaryngol Clin North Am. 2002, 35(4):711-732.

4. Shahnaz N. Multi-frequency tympanometry and evidencebased pratice. ASHA Perspective on Hearing and Hearing Disorders: Research and diagnosis. 2007, 11(1):2-12.

5. Lai D, Li W, Xian J, Liu S. Multifrequency tympanometry in adults with otitis media with efusion. Eur Arch Otorhinolaryngol. 2008, 265(9): 1021-1025.

6. Petrak M. Tympanometry beyond $226 \mathrm{~Hz}-$ What is different in babies? [texto na internet]. Audiology. 18 nov 2002. [acesso em 2009 mar 17]. Disponível em: http:// www.audiologyonline.com

7. Palmu AA, Syrjänen R. Diagnostic value of tympanometry using subject-specific normative values. Int J Pediatr Otorhinolaryngol. 2005, 69(7):965-971.

8. Smith CG, Paradise JL, Sabo DL, Rockette HE, Kurs-Lasky M, Bernard BS, et al. Tympanometric findings and the probability of middle ear effusion in 3686 infants and young children. Pediatrics. 2006, 118(1):1-13.

9. Feniman MR, Souza AG, Jorge JC, Lauris JRP. Achados otoscópicos e timpanométricos em lactentes com fissura labiopalatina. Rev Bras Otorrinolaringol. 2008, 74(2):248252.

10. Melo JJ, Lewis DR, Marchiori LLM. Resultados timpanométricos: lactentes de seis meses de idade. Rev CEFAC. 2008, 10(1):104-109.

11. De Ceulaer G, Somers T, Offeciers FE, Govaerts PJE. Middle ear status in ears showing high admittance tympanograms. Clinical value of the use of the two component $678 \mathrm{~Hz}$ otoadmittance tympanogram. Acta Otorhinolaryngol Belg. 2002, 56(3):313-317.

12. Margolis RH, Bass-Ringdahl S, Hanks WD, Holte L, Zapala D. Tympanometry in newborn infants - $1 \mathrm{kHz}$ norms. J Am Acad Audiol. 2003, 14(7):383-392.

13. Baldwin M. Choice of probe tone and classification of trace patterns in tympanometry undertaken in early infancy. Int Audiol. 2006, 45(7):417-427.

14. Linares AE, Carvallo RMM. Medidas imitanciométricas em crianças com ausência de emissões otoacústicas. Rev Bras Otorrinolaringol. 2008, 74(3):410-416.

15. Swanepoel DW, Werner S, Hugo R, Louw B, Owen Rina, Swanepoel A. High frequency immittance for neonates: a normative study. Acta Oto-Laryngol. 2007, 127(1):49-56. 
16. Joint Committee on Infant Hearing. Year 2007 position statement: principles and guidelines for early hearing. Pediatrics. 2007, 120(4):898-921.

17. Grason-Stadler. GSI Tympstar version 2 - Middle ear analyzer: Reference Instruction Manual. $9^{\text {th }}$ ed. Madison: Grason-Stadler; 2005.

18. Sutton G, Baldwin M, Brooks D, Gravel J, Thornton R. Tympanometry in neonates and infants under 4 months: a recommended test protocol. 2002. [acesso em 2009 jun 14]. Disponivel em: http://www.nhsp.info/ getdata.php?id=135.

19. Carvallo RMM. Medidas de imitância acústica em crianças de zero a oito meses de idade [tese]. São Paulo(SP): Escola Paulista de Medicina;1992.

20. Cazelatto AS, Collela-Santos MF. Imitanciometria em lactentes com tom de sonda de 226 e 1000 Hertz. In: Anais do $16^{\circ}$ Congresso Interno de Iniciação Científica; 2008; Campinas, SP. Campinas: Unicamp, 2008.

21. Macedo CC. Timpanometria em lactentes com fissure labiopalatina utilizando sonda de multifrequência [dissertação]. Bauru (SP): Hospital de Reabilitação de Anomalias Craniofaciais; 2010.

22. Margolis RH. Tympanometry in infants. State of the art. In: Harford ER, Bess FH, Bluestone CD, Klein JO. Impedance screening for middle ear diseases in children. $1^{\text {st }}$ ed. New York: Grunfe \& Stratton; 1978. p. 41-56.

23. Shanks JE. Tympanometry. Earand Hear. 1984, 5(5):268298.

24. Isaac ML, Oliveira JAA, Holanda F. Importância da otomicroscopia e imitanciometria na detecção precoce de efusão no ouvido médio de crianças assintomáticas em ambulatório de puericultura. Rev Bras Otorrinolaringol. 1999, 65(2):122-127.

25. Kei J, Allison-Levick J, Dockray J, Harrys R, Kirkegard C, Wong J,et al. High-frequency (1000 Hz) tympanometry in normal neonates. J Am Acad Audiol. 2003, 14(1):20-28.

26. Alaerts J, Luts H, Wouters J. Evaluation of middle ear function in young children: clinical guidelines for use of 226and 1.000-Hz tympanometry. Otol Neurotol. 2007, 28(6):727-723.

27. Engel J, Anteunis L, Chenault M, Marres E. Otoscopic findings in relation to tympanometry during infancy. Eur Arch Otorhinolaryngol. 2000, 257(7):366-371.
28. Silva KAL, Novaes BACC, Lewis DR, Carvallo RMM. Achados timpanométricos em neonatos com emissões otoacústicas presentes: medidas e interpretações. Rev Bras Otorrinolaringol. 2007, 73(5):633-639.

29. Swanepoel DW, Hugo R, Louw B. Infant hearing screening at immunization clinics in South Africa. J Pediatr Otorhinolaryngol. 2006, 70(7):1241-1249.

30. Van Rooyen S. Immitance in infants 0-12 months: measurements using a $1000 \mathrm{~Hz}$ probe tone [tese na internet]. Pretoria:África do Sul. University of Pretoria; 2006. [acesso em 2010 fev 25]. Disponível em: http://upetd.up.ac.za/ thesis/available/etd-10292007-142816/unrestricted/ Oofront.pdf

31. Rhodes MC, Margolis RH, Hirsch JE, Napp AP. Hearing screening in the newborn intensive care nursery: comparison of methods. Otolaryngol Head Neck Surg. 1999, 120(6):799808 .

32. Calandruccio L, Fitzgerald TS, Prieve BA. Normative multifrequency tympanometry in infants and toddlers. J Am Acad Audiol. 2006, 17(7):470-480.

33. Jerger JF. Clinical experience with impedance audiometry. Arch Otolaryngol. 1970, 92(4):311-324.

34. Hall III JW, Chandler D. Timpanometria na audiologia clínica. In: Katz, J. Tratado de audiologia clínica. 4ª ed. São Paulo: Manole; 1999. p. 281-297.

35. Prieve BA, Calandruccio L, Fitzgerald T, Mazevski L, Georgantas LM. Changes in transient-evoked otoacoustic emission levels with negative tympanometric peak pressure in infants and toddlers. Ear Hear. 2008, 29(4):533-542.

36. Margolis RH, Heller JW. Screening tympanometry: criteria for medical referral. Audiology. 1987, 26:190-208.

37. Shanks JE, Stelmachowicz PG, Beauchaine KL, Schulte L. Equivalent ear canal volumes in children pre- and postympanostomy tube insertion. J Speech Hear Research. 1992, 35:936-941.

38. Mazlan R, Kei J, Hickson L, Stapleton C, Grant S, Lim S, et al. High frequency immittance findings: Newborn versus six-week-old infants. Int J Audiol. 2007, 46(11):711-717.

39. Grason-Stadler. Instruction manual GSI, version 2 -Middle ear analyzer. $4^{\text {th }}$ ed. Massachussets: Grason-Stadler; 1994.

40. Fowler CG, Shanks JE. Tympanometry. In: Katz J. Handbook of clinical audiology. $5^{\text {th }}$ ed. Philadelphia: Lippincott Willians \& Willians; 2002. p.175-204. 
41. Mazlan R, Kei J, Hickson L, Gavranich J, Linning R. Testretest reproducibility of the $1.000 \mathrm{~Hz}$ tympanometry test in newborn and six-week-old healthy infants. Int J Audiol: Early Online. 2010. [acesso em 2010 set 22]; 0(0):1-8. Disponível em : http://informahealthcare.com/doi/abs/ $10.3109 / 14992027.2010 .493182$

42. Piazentin-Penna SHA. Identificação auditiva em crianças de 3 a 12 meses de idade com fissura labiopalatina [tese].Bauru (SP): Hospital de Reabilitação de Anomalias Craniofaciais; 2002.

43. Garcia MV, Azevedo MF, Testa JR. Medidas de imitância acústica em lactentes com 226 Hz e 1000 Hz: correlação com as emissões otoacústicas e o exame otoscópico. Rev Bras Otorrinolaringol. 2009, 75(1):80-89.

44. Harris PK, Hurchinson KM, Moravec J. The use of tympanometry and pneumatic otoscopy for predicting middle ear disease. Am J Audiol. 2003, 14(1): 3-13.

45. Wimmer E, Toleti B, Berghaus A, Baumann U, Nejedlo, I. Impedance audiometry in infants with cleft palate: the standard $226 \mathrm{~Hz}$ probe tone has no predictive value for the middle ear condition. Int J Pediatr Otorhinolaryngol. 2010, 74(6):586-590 